\title{
Article \\ Efficient Splitting Methods for Solving Tensor Absolute Value Equation
}

\author{
Jing Ning ${ }^{1}$, Yajun $\mathrm{Xie}^{2, *}$ and Jie Yao ${ }^{2}$ \\ 1 College of Computer and Information Sciences, Fujian Agriculture and Forestry University, \\ Fuzhou 350108, China; ningjing@fafu.edu.cn \\ 2 School of Big Data, Fuzhou University of International Studies and Trade, Fuzhou 350202, China; \\ yj@fzfu.edu.cn \\ * Correspondence: xyj@fzfu.edu.cn
}

check for updates

Citation: Ning, J.; Xie, Y.; Yao, J. Efficient Splitting Methods for Solving Tensor Absolute Value Equation. Symmetry 2022, 14, 387. https://doi.org/10.3390/ sym 14020387

Academic Editors: Juan Luis García Guirao and Calogero Vetro

Received: 29 December 2021 Accepted: 8 February 2022

Published: 15 February 2022

Publisher's Note: MDPI stays neutral with regard to jurisdictional claims in published maps and institutional affiliations.

Copyright: (c) 2022 by the authors. Licensee MDPI, Basel, Switzerland. This article is an open access article distributed under the terms and conditions of the Creative Commons Attribution (CC BY) license (https:// creativecommons.org/licenses/by/ $4.0 /)$.
Abstract: The tensor absolute value equation is a class of interesting structured multilinear systems. In this article, from the perspective of pure numerical algebra, we first consider a tensor-type successive over-relaxation method (SOR) (called TSOR) and tensor-type accelerated over-relaxation method (AOR) (called TAOR) for solving tensor absolute value equations. Furthermore, one type of preconditioned tensor splitting method is also applied for solving structured multilinear systems. Numerical experiments adequately demonstrate the efficiency of the presented methods.

Keywords: tensor absolute value equation (TAVE); splitting iteration; successive over-relaxation method (SOR); accelerated over-relaxation method (AOR); preconditioned technique

\section{Introduction}

In this paper, we focus on the following tensor absolute value equation (TAVE):

$$
\mathcal{A} x^{m-1}-|x|^{[m-1]}=b,
$$

where $\mathcal{A}=\left(a_{i_{1} \cdots i_{m}}\right) \in \mathbb{R}^{[m, n]}$ is an order $m$ dimension $n$ tensor, vector $b \in \mathbb{R}^{n}$ and $|x|^{[m-1]}$ is dimension $n$ with the following:

$$
|x|^{[m-1]}:=\left(\left|x_{1}\right|^{m-1},\left|x_{2}\right|^{m-1}, \cdots,\left|x_{n}\right|^{m-1}\right)^{T} \in \mathbb{R}^{n},
$$

and $x \in \mathbb{R}^{n}$ is an undetermined vector.

It is obvious that (1) will reduce to the well-known absolute value equation (AVE) as follows:

$$
A x-|x|=b,
$$

when $m=2$, and $A$ is a matrix with appropriate dimensions. Formula (1), in fact, can also be regarded as multilinear systems:

$$
\mathcal{F} x^{m-1}=b
$$

with special structure $\mathcal{F}=\mathcal{A}-D_{x} \cdot \mathcal{I}_{m}$, where the following is the case.

$$
D_{x}=\operatorname{diag}(\operatorname{sgn}(x)) \in \mathbb{R}^{n \times n}, \mathcal{I}_{m} \in \mathbb{R}^{[m, n]} .
$$

Symbol '.' denotes a matrix-tensor product (see details in the next section).

An essential problem in pure and applied mathematics is solving various linear or nonlinear systems. The rapid and efficient techniques of solution-finding for multilinear systems [1-6]) are becoming increasingly significant in the field of science and engineering 
due to their extensive applications. In particular, some approaches with respect to higher order tensors [7-15] for data analysis have been widely studied in the era of Big Data.

Song and Qi [16] introduced a class of complementarity problems, named tensor complementarity problems. It was testified that the well-known absolute value equation (AVE) [17] is equivalent to a generalized linear complementarity problem [18] (see $[19,20]$ for more details).

Normally, it is hard to obtain the exact solution by means of direct methods even for smaller-scale general linear systems, which greatly urges substantial developments of presenting various iterative strategies. Many research works have been investigated fast solvers for multilinear systems. Ding and Wei [21,22] proposed some classical iteratives, such as Jacobi, Gauss-Seidel methods and Newton methods by translating (4) into an optimization problem. In general, the computational cost for the Newton method is expensive on account of the inverse of the matrix involved. Then, Han [23] investigated a homotopy method by the Euler-Newton prediction-correction technique to solve multilinear systems with nonsymmetric $\mathcal{M}$-tensors, which demonstrated a better result than the Newton method in the sense of convergence performances. The tensor splitting method and its convergence results have been studied by Liu and Li et al. [24]. Furthermore, some comparison results for splitting iteration for solving multilinear systems were investigated widely in $[25,26]$. It is observed that the results of studies about tensor absolute value equation (TAVE) (1) are relatively rare in the existing literature. Du et al. presented the tensor absolute value equation (TAVE) [27] and introduced the Levenberg-Marquardt method for solving this (1) from the point of an optimization theory. Ling et al. [28] provided some conditions for the existence of solutions of TAVE with the help of degree theory. Moreover, by fixed point theory, the system of TAVE has at least one solution under some workable assumptions.

Fixed-point typed methods and splitting tricks can be applicable for the consideration of some problems with low-rank tensor structure, e.g., the dynamical or steady-state multicomponent coagulation equation [29,30]. Recently, a novel approach was proposed to solve TAVE, where the interval tensor is introduced, which is the main tool for proving the unique solvability of TAVE. Then, a sufficient condition for TAVE was presented to obtain a unique solution [31]. TAVE can be related to global optimization via tensor-formats, e.g., tensor train [32]. In this case, the tensor-matrix can be considered as a diagonal, and the global maximum is exactly the largest eigenvalue of such diagonalized tensor. To our knowledge, there have been not many research works for solving TAVE from the splitting iteration perspective. Based on this consideration, some efficient splitting iteration methods will be studied to solve TAVE. Tensor-type successive overrelaxation method (TSOR) and tensor-type accelerated overrelaxation method (TAOR) are intensively surveyed in this paper. Furthermore, a preconditioner is also constructed ultimately for solving TAVE (1).

The remainder of this paper is organized as follows. In Section 2, some basic and useful notations are described simply. In Section 3, we will propose tensor splitting iteration schemes TAOR and TSOR for solving TAVE. Meanwhile, the analyses of convergence conditions are established in detail. Then, some numerical experiments are provided in Section 4 to illustrate the superiority of the presented iteration methods. Finally, a conclusion is provided in Section 5.

\section{Preliminaries}

Let $A \in \mathbb{R}^{[2, n]}$ and $B \in \mathbb{R}^{[k, n]}$. The matrix-tensor product $\mathcal{C}=A \cdot \mathcal{B} \in \mathbb{R}^{[k, n]}$ is defined by the following.

$$
c_{j i_{2} \cdots i_{k}}=\sum_{j_{2}=1}^{n} a_{j j_{2}} b_{j_{2} i_{2} \cdots i_{k}} .
$$


The above formula can be regarded as follows:

$$
\mathcal{C}_{(1)}=(A \cdot \mathcal{B})_{(1)}=A \mathcal{B}_{(1)}
$$

where $\mathcal{C}_{(1)}$ and $\mathcal{B}_{(1)}$ are the matrices generated from $\mathcal{C}$ and $\mathcal{B}$ flattened along first index. For more details, see $[33,34]$.

Definition 1. (In [35]). Let $\mathcal{A}=\left(a_{i_{1} i_{2} \cdots i_{m}}\right) \in \mathbb{R}^{[m, n]}$. Then, the majorization matrix $M(\mathcal{A})$ of $\mathcal{A}$ is the $n \times n$ matrix with the entries.

$$
M(\mathcal{A})_{i j}=a_{i j \ldots j}, \quad i, j=1,2, \cdots, n
$$

Definition 2. (In [25]). Let $\mathcal{A}=\left(a_{i_{1} i_{2} \cdots i_{m}}\right) \in \mathbb{R}^{[m, n]}$. If $M(\mathcal{A})$ is a nonsingular matrix and $\mathcal{A}=$ $M(\mathcal{A}) \mathcal{I}_{m}$, we call $M(\mathcal{A})^{-1}$ the order-2 left-inverse of tensor $\mathcal{A}$, and $\mathcal{A}$ is called left-nonsingularity, where $\mathcal{I}_{m}$ is an identity tensor with all diagonal elements being 1 .

Definition 3. (In [25]). Let $\mathcal{A}, \mathcal{E}, \mathcal{F} \in \mathbb{R}^{[m, n]} . \mathcal{A}=\mathcal{E}-\mathcal{F}$ is named as a splitting of tensor $\mathcal{A}$ if $\mathcal{E}$ is left-nonsingular. A regular splitting of $\mathcal{A}$ if $\mathcal{E}$ is left-nonsingular with $M(\mathcal{E})^{-1} \geq 0$ and $\mathcal{F} \geq 0$ (here, $\leq$ or $\geq$ denotes elementwise). A weak regular splitting of $\mathcal{A}$ if $\mathcal{E}$ is left-nonsingular with $M(\mathcal{E})^{-1} \mathcal{F} \geq 0$. A convergence splitting occurrs if the spectral radius of $M(\mathcal{E})^{-1} \mathcal{F}$ is less than 1, i.e., $\rho\left(M(\mathcal{E})^{-1} \mathcal{F}\right)<1$.

Definition 4. (In [36]). Let $\mathcal{A}=\left(a_{i_{1} i_{2} \cdots i_{m}}\right) \in \mathbb{R}^{[m, n]}$. A pair $(\lambda, x) \in \mathbb{C} \times\left(\mathbb{C}^{n} \backslash\{0\}\right)$ is called an eigenvalue-eigenvector of tensor $\mathcal{A}$ if they satisfy the following systems:

$$
\mathcal{A} x^{m-1}=\lambda x^{[m-1]}
$$

where $x^{[m-1]}=\left(x_{1}^{m-1}, x_{2}^{m-1}, \cdots, x_{n}^{m-1}\right)^{T} .(\lambda, x)$ is named as an H-eigenpair if both $\lambda$ and vector $x$ are real.

Definition 5. Let $\rho(A)=\max \{|\lambda| \mid \lambda \in \sigma(A)\}$ be the spectral radius of $\mathcal{A}$, where $\sigma(\mathcal{A})$ is the set of all eigenvalues of $\mathcal{A}$.

Definition 6. (In [37]). Let $\mathcal{A}=\left(a_{i_{1} i_{2} \cdots i_{m}}\right) \in \mathbb{R}^{[m, n]}$. $\mathcal{A}$ is called a Z-tensor if its off-diagonal entries are non-positive. $\mathcal{A}$ is called an $M$-tensor if there exists a non-negative tensor $\mathcal{B}$ and $a$ positive real number $\eta \geq \rho(B)$ such that

$$
\mathcal{A}=\eta \mathcal{I}_{m}-\mathcal{B}
$$

If $\eta>\rho(\mathcal{B})$, then $\mathcal{A}$ is called a strong $M$-tensor.

\section{Reformulation and Tensor Splitting Iteration}

We first transform the TAVE (1) into the equivalent form.

$$
\left\{\begin{array}{l}
\mathcal{A} x^{m-1}-\mathcal{I}_{m} y^{m-1}=b \\
-|x|^{[m-1]}+\mathcal{I}_{m} y^{m-1}=0
\end{array}\right.
$$

In other words, we have the following:

$$
H z=f \text {, }
$$

where

$$
H=\left(\begin{array}{cc}
M(\mathcal{A}) & -M\left(\mathcal{I}_{m}\right) \\
-D_{x} & M\left(\mathcal{I}_{m}\right)
\end{array}\right) \in \mathbb{R}^{2 n \times 2 n}, z=\left(\begin{array}{c}
\mathcal{I}_{m} x^{m-1} \\
\mathcal{I}_{m} y^{m-1}
\end{array}\right) \in \mathbb{R}^{2 n}, f=\left(\begin{array}{c}
b \\
0
\end{array}\right) \in \mathbb{R}^{2 n}
$$


$\mathcal{I}_{m}$ is an m-order n-dimensional unit tensor for which its entries are 1 if and only if $i_{1}=\cdots=i_{m}$ and otherwise zero, $M(\cdot)$ is the majorization matrix in Definition 1 and symbolic matrix $D_{x}$ is defined by (5).

If we consider the tensor splitting $[38,39]$ :

$$
\mathcal{B}=\mathcal{E}-\mathcal{F}
$$

then, it is easy to briefly establish an iterative method for solving multi-linear systems

$$
\mathcal{B} x^{m-1}=b .
$$

Clearly, the above multi-linear systems can be written as follows.

$$
\mathcal{E} x^{m-1}=\mathcal{F} x^{m-1}+b,
$$

In other words, we have the following.

$$
\mathcal{I}_{m} x^{m-1}=M(\mathcal{E})^{-1} \mathcal{F} x^{m-1}+M(\mathcal{E})^{-1} b,
$$

Here, we use the property of two-order left-nonsingularity of tensor $\mathcal{E}$, and $\mathcal{I}_{m}$ is an identify tensor with appropriate orders.

Moreover, it conceives the following splitting:

$$
\mathcal{A}=\mathcal{A}_{D}-\mathcal{A}_{L}-\mathcal{A}_{U},
$$

where $\mathcal{A}_{D}$ is the diagonal tensor, and $\mathcal{A}_{L}$ and $\mathcal{A}_{U}$ are strictly lower and upper triangular tensors generated by tensor $\mathcal{A}$ in (10) [25].

If we consider the TAOR iterative method for (11), it gives rise to the following:

$$
\left(H_{D}-\sigma H_{L}\right) z_{(k+1)}=\left[(1-\omega) H_{D}+(\omega-\sigma) H_{L}+\omega H_{U}\right] z_{(k)}+\omega f,
$$

where parameters satisfy $0<\omega<2, \sigma>0$ and the following.

$$
H_{D}=\left(\begin{array}{cc}
M\left(\mathcal{A}_{D}\right) & 0 \\
0 & M\left(\mathcal{I}_{m}\right)
\end{array}\right), H_{L}=\left(\begin{array}{cc}
M\left(\mathcal{A}_{L}\right) & 0 \\
D_{x} & 0
\end{array}\right), H_{U}=\left(\begin{array}{cc}
M\left(\mathcal{A}_{U}\right) & M\left(\mathcal{I}_{m}\right) \\
0 & 0
\end{array}\right) .
$$

Thus, we have the following:

$$
\begin{aligned}
& \left(\begin{array}{cc}
M\left(\mathcal{A}_{D}\right)-\sigma M\left(\mathcal{A}_{L}\right) & 0 \\
-\sigma D_{x} & M\left(\mathcal{I}_{m}\right)
\end{array}\right)\left(\begin{array}{c}
\mathcal{I}_{m} x_{(k+1)}^{m-1} \\
\mathcal{I}_{m} y_{(k+1)}^{m-1}
\end{array}\right) \\
& =\left(\begin{array}{cc}
(1-\omega) M\left(\mathcal{A}_{D}\right)+(\omega-\sigma) M\left(\mathcal{A}_{L}\right)+\omega M\left(\mathcal{A}_{U}\right) & \omega M\left(\mathcal{I}_{m}\right) \\
(\omega-\sigma) D_{x} & (1-\omega) M\left(\mathcal{I}_{m}\right)
\end{array}\right)\left(\begin{array}{l}
\mathcal{I}_{m} x_{(k)}^{m-1} \\
\mathcal{I}_{m} y_{(k)}^{m-1}
\end{array}\right)+\omega f,
\end{aligned}
$$

which can be written as follows.

$$
\left\{\begin{array}{l}
\mathcal{I}_{m} x_{(k+1)}^{m-1}=\left[M\left(\mathcal{A}_{D}-\sigma \mathcal{A}_{L}\right)\right]^{-1}\left\{\left[(1-\omega) \mathcal{A}_{D}+(\omega-\sigma) \mathcal{A}_{L}+\omega \mathcal{A}_{U}\right] x_{(k)}^{m-1}+\omega\left(\mathcal{I}_{m} y_{(k)}^{m-1}+b\right)\right\}, \\
\mathcal{I}_{m} y_{(k+1)}^{m-1}=(\omega-\sigma)\left|x_{k}\right|^{[m-1]}+(1-\omega) \mathcal{I}_{m} y_{(k)}^{m-1}+\sigma\left|x_{k+1}\right|^{[m-1]} .
\end{array}\right.
$$

Based on the above analysis, the following Algorithm 1 is proposed formally. 
Algorithm 1 Tensor AOR splitting iterative method (TAOR) for TAVE

Step 1 Input vector $b$, tensor $\mathcal{A}$. Given a precision $\varepsilon>0$, parameters $0<\omega<2, \sigma>0$, and initial vector $x_{0}, y_{0}$. Set $k:=0$.

Step 2 If $\left\|\mathcal{A} x_{(k)}^{m-1}-\left|x_{(k)}\right|^{[m-1]}-b\right\|_{2}<\varepsilon$ stop; otherwise, go to Step 3 .

Step 3 Compute iterative schemes

$$
\left\{\begin{array}{l}
x_{(k+1)}=\left\{\left[M\left(\mathcal{A}_{D}-\sigma \mathcal{A}_{L}\right)\right]^{-1}\left\{\left[(1-\omega) \mathcal{A}_{D}+(\omega-\sigma) \mathcal{A}_{L}+\omega \mathcal{A}_{L}\right] x^{m-1}+\omega\left(\mathcal{I}_{m} y_{(k)}^{m-1}+b\right)\right\}\right\}^{\left[\frac{1}{m-1}\right]}, \\
y_{(k+1)}=\left\{(\omega-\sigma)\left|x_{(k)}\right|^{[m-1]}+(1-\omega) \mathcal{I}_{m} y_{(k)}^{m-1}+\sigma\left|x_{(k+1)}\right|^{[m-1]}\right\}^{\left[\frac{1}{m-1}\right]} .
\end{array}\right.
$$

Step 4 Set $k:=k+1$, return to Step 2 .

Remark 1. If we take $\sigma=\omega$ in (17), the TAOR method will be reduced to the TSOR method with the following iterative schemes.

$$
\left\{\begin{array}{l}
x_{(k+1)}=\left\{\left[M\left(\mathcal{A}_{D}-\omega \mathcal{A}_{L}\right)\right]^{-1}\left\{\left[(1-\omega) \mathcal{A}_{D}+\omega \mathcal{A}_{U}\right] x_{(k)}^{m-1}+\omega\left(\mathcal{I}_{m} y_{(k)}^{m-1}+b\right)\right\}\right\}^{\left[\frac{1}{m-1}\right]}, \\
y_{(k+1)}=\left\{(1-\omega) \mathcal{I}_{m} y_{(k)}^{m-1}+\omega\left|x_{(k+1)}\right|^{[m-1]}\right\}^{\left[\frac{1}{m-1}\right]} .
\end{array}\right.
$$

Remark 2. Furthermore, let us consider a preconditioned TAVE:

$$
P \cdot \mathcal{A} x^{m-1}=P c,
$$

where $c=\mathcal{I}_{m} y_{(k)}^{m-1}+b$. It follows from the following splitting:

$$
\begin{aligned}
\hat{\mathcal{A}}: & =P \cdot \mathcal{A} \\
& =P \cdot \mathcal{A}_{D}-P \cdot \mathcal{A}_{L}-P \cdot \mathcal{A}_{U} \\
& =\hat{\mathcal{A}}_{D}-\hat{\mathcal{A}}_{L}-\hat{\mathcal{A}}_{U}
\end{aligned}
$$

that the following is the case.

$$
\left\{\begin{array}{l}
x_{(k+1)}=\left\{\left[M\left(\hat{\mathcal{A}}_{D}-\omega \hat{\mathcal{A}}_{L}\right)\right]^{-1}\left\{\left[(1-\omega) \hat{\mathcal{A}}_{D}+\omega \hat{\mathcal{A}}_{U}\right] x_{(k)}^{m-1}+\omega P\left(\mathcal{I}_{m} y_{(k)}^{m-1}+b\right)\right\}\right\}^{\left[\frac{1}{m-1}\right]}, \\
y_{(k+1)}=\left\{(1-\omega) \mathcal{I}_{m} y_{(k)}^{m-1}+\omega \mid x_{(k+1)}{ }^{[m-1]}\right\}^{\left[\frac{1}{m-1}\right]} .
\end{array}\right.
$$

In the numerical experiment section, a type of preconditioner will be introduced to further accelerate Algorithm 1.

Next, we will provide some critical lemmas [21,27] about the existence of solution for TAVE.

Lemma 1. Let $\mathcal{A}=\left(a_{i_{1} \ldots i_{m}}\right) \in \mathbb{R}^{[m, n]}$. If $\mathcal{A}$ is a strong $\mathcal{M}$-tensor, then for every positive vector $b$, the multilinear system of equations $\mathcal{A} x^{m-1}=b$ has a unique positive solution.

Lemma 2. Let $\mathcal{A}=\left(a_{i_{1} \cdots i_{m}}\right) \in \mathbb{R}^{[m, n]}$ be a $\mathcal{Z}$-tensor. Then, it is a strong $\mathcal{M}$-tensor if and only if the multilinear system of equations $\mathcal{A} x^{m-1}=b$ has a unique positive solution for every positive vector $b$.

Lemma 3. Let $\mathcal{A}=\left(a_{i_{1} \cdots i_{m}}\right) \in \mathbb{R}^{[m, n]}$ be an $\mathcal{M}$-tensor and $b \geq 0$. If there exists $v \geq 0$ such that $\mathcal{A} v^{m-1} \geq b$, then the multilinear system of equations $\mathcal{A} x^{m-1}=b$ has a non-negative solution.

Lemma 4. Let $\mathcal{A}=\left(a_{i_{1} \ldots i_{m}}\right) \in \mathbb{R}^{[m, n]}$. If $\mathcal{A}$ can be written as $\mathcal{A}=\eta \mathcal{I}-\mathcal{B}$ with $\mathcal{B} \geq 0$ and $\eta \geq \rho(\mathcal{B})+1$, then for every positive vector $b$, TAVE (10) has a unique positive solution. 
Lemma 5. Let the real quadratic equation $x^{2}-p x+q=0$, where $p$ and $q$ are real numbers. Both roots of the real quadratic equation are less than one in modulus if and only if $|q|<1$ and $|p|<1+q$.

For the sake of convergence analysis, it is necessary to provide the definition of iterative errors as follows.

$$
\begin{aligned}
e_{(k)}^{x}: & =\mathcal{I}_{m} x_{*}^{m-1}-\mathcal{I}_{m} x_{(k)}^{m-1} \\
& =x_{*}^{[m-1]}-x_{(k)}^{[m-1]}, \\
e_{(k)}^{y}: & =\mathcal{I}_{m} y_{*}^{m-1}-\mathcal{I}_{m} y_{(k)}^{m-1} \\
& =y_{*}^{[m-1]}-y_{(k)}^{[m-1]} .
\end{aligned}
$$

Theorem 1. Let $\mathcal{A}=\left(a_{i_{1} \cdots i_{m}}\right) \in \mathbb{R}^{[m, n]}$. If $\mathcal{A}$ can be written as $\mathcal{A}=\eta \mathcal{I}-\mathcal{B}$ with $\mathcal{B} \geq 0$ and $\eta \geq \rho(\mathcal{B})+1, b>0$, then the iterative sequence $\left\{x_{(k)}\right\}(k=0,1, \cdots)$ generated by the Algorithm 1 converges to a unique positive solution $x_{*}$ of the TAVE (10) if the following is the case:

$$
\left|\kappa \theta_{1}-\theta_{2}\right|<1,\left|\kappa+\theta_{1}+\theta_{3}\right|<1+\kappa \theta_{1}-\theta_{2}
$$

where

$$
\theta_{1}=|1-\omega|, \theta_{2}=|\omega-\sigma|, \theta_{3}=\omega \sigma \rho
$$

and the following is obtained.

$$
\begin{gathered}
\kappa=\left\|\left[M\left(\mathcal{A}_{D}-\sigma \mathcal{A}_{L}\right)\right]^{-1}\left[(1-\omega) M\left(\mathcal{A}_{D}\right)+(\omega-\sigma) M\left(\mathcal{A}_{L}\right)+\omega M\left(\mathcal{A}_{U}\right)\right]\right\|, \\
\rho=\left\|\left[M\left(\mathcal{A}_{D}-\sigma \mathcal{A}_{L}\right)\right]^{-1}\right\| .
\end{gathered}
$$

Proof. Clearly, the existence of solution $x_{*}$ for TAVE is guaranteed by Lemmas 1-4. From (18)-(20), it generates the following.

$$
\left\{\begin{aligned}
e_{(k+1)}^{x} & =\left[M\left(\mathcal{A}_{D}-\sigma \mathcal{A}_{L}\right)\right]^{-1}\left\{\left[(1-\omega) \mathcal{A}_{D}+(\omega-\sigma) \mathcal{A}_{L}+\omega \mathcal{A}_{U}\right] e_{(k)}^{x}+\omega e_{(k)}^{y}\right\} \\
e_{(k+1)}^{y} & =(\omega-\sigma)\left(\left|x_{*}\right|^{[m-1]}-\left|x_{(k)}\right|^{[m-1]}\right)+(1-\omega) e_{(k)}^{y}+\sigma\left(\left|x_{*}\right|^{[m-1]}-\left|x_{(k+1)}\right|^{[m-1]}\right) .
\end{aligned}\right.
$$

By (25), we have the following.

$$
\begin{gathered}
\left\|e_{(k+1)}^{x}\right\|=\|\left[M\left(\mathcal{A}_{D}-\sigma \mathcal{A}_{L}\right)\right]^{-1}\left[(1-\omega) M\left(\mathcal{A}_{D}\right)+(\omega-\sigma) M\left(\mathcal{A}_{L}\right)\right. \\
\left.\quad+\omega M\left(\mathcal{A}_{U}\right)\right]\|\| e_{(k)}^{x}\|+\omega\|\left[M\left(\mathcal{A}_{D}-\sigma \mathcal{A}_{L}\right)\right]^{-1}\|\| e_{(k)}^{y} \| \\
=\kappa\left\|e_{(k)}^{x}\right\|+\omega \rho\left\|e_{(k)}^{y}\right\|, \\
\left\|e_{(k+1)}^{y} \leq \leq|\omega-\sigma|\right\|\left|x_{*}^{[m-1]}\right|-\left|x_{(k)}^{[m-1]}\right|\|+|1-\omega|\| e_{(k)}^{y}\|+\sigma\|\left|x_{*}^{[m-1]}\right|-\left|x_{(k+1)}^{[m-1]}\right| \| \\
\leq|\omega-\sigma|\left\|e_{(k)}^{x}\right\|+|1-\omega|\left\|e_{(k)}^{y}\right\|+\sigma\left\|e_{(k+1)}^{x}\right\| .
\end{gathered}
$$

Based on (26) and (27), it can be reformulated as follows.

$$
\left\|e_{(k+1)}^{y}\right\| \leq(|\omega-\sigma|+\sigma \kappa)\left\|e_{(k)}^{x}\right\|+(|1-\omega|+\sigma \omega \rho)\left\|e_{(k)}^{y}\right\| .
$$


It follows from (26) and (28) that the following is the case.

$$
\begin{aligned}
\left(\begin{array}{c}
\left\|e_{(k+1)}^{x}\right\| \\
\left\|e_{(k+1)}^{y}\right\|
\end{array}\right) & \leq\left(\begin{array}{cc}
\kappa & \omega \rho \\
|\omega-\sigma|+\sigma \kappa & |1-\omega|+\sigma \omega \rho
\end{array}\right)\left(\begin{array}{c}
\left\|e_{(k)}^{x}\right\| \\
\left\|e_{(k)}^{y}\right\|
\end{array}\right) \\
\leq \cdots & \leq\left(\begin{array}{cc}
\kappa & \omega \rho \\
|\omega-\sigma|+\sigma \kappa & |1-\omega|+\sigma \omega \rho
\end{array}\right)^{k+1}\left(\begin{array}{c}
\left\|e_{(0)}^{x}\right\| \\
\left\|e_{(0)}^{y}\right\|
\end{array}\right) .
\end{aligned}
$$

Let the following be the case.

$$
G=\left(\begin{array}{cc}
\kappa & \omega \rho \\
|\omega-\sigma|+\sigma \kappa & |1-\omega|+\sigma \omega \rho
\end{array}\right) .
$$

When $\rho(G)<1$, it must be $\lim _{k \rightarrow \infty} G^{k+1}=0$. That is, the following is the case:

$$
\lim _{k \rightarrow \infty}\left\|e_{(k)}^{x}\right\|=0, \quad \lim _{k \rightarrow \infty}\left\|e_{(k)}^{y}\right\|=0
$$

In other words, we have the following.

$$
\lim _{k \rightarrow \infty} x_{(k)}=x_{*}, \lim _{k \rightarrow \infty} y_{(k)}=y_{*}
$$

Now, we should focus on inequality $\rho(G)<1$. First let $(\lambda, v)$ be an eigen-pair of matrix $G$. The eigenvalue equation is as follows:

$$
G v=\lambda v,
$$

and it implies the following:

$$
\left\{\begin{array}{l}
k v_{1}+\omega \rho v_{2}=\lambda v_{1} \\
(|\omega-\sigma|+\sigma \kappa) v_{1}+(|1-\omega|+\sigma \omega \rho) v_{2}=\lambda v_{2}
\end{array}\right.
$$

where $v=\left(v_{1}^{T}, v_{2}^{T},\right)^{T}$. By some calculations, it generates the following.

$$
\lambda^{2}-[\kappa+|1-\omega|+\omega \sigma \rho] \lambda+\kappa|1-\omega|-|\omega-\sigma|=0 .
$$

According to Lemma 5, all eigenvalues $\lambda$ are less than one in modulus if and only if the following is the case.

$$
\left|\kappa \theta_{1}-\theta_{2}\right|<1,\left|\kappa+\theta_{1}+\theta_{3}\right|<1+\kappa \theta_{1}-\theta_{2} .
$$

This completes the proof.

\section{Numerical Experiments}

In this section, some numerical examples are discussed to validate the performance of the effectiveness of the proposed tensor AOR method ('TAOR') (Algorithm 1) and tensor inexact Levenberg-Marquardt-type method (TILM) (see [27]) for solving the tensor absolute value equation. We compare their performances of convergence by the iteration step (denoted as 'IT'), elapsed CPU time in seconds (denoted as 'CPU') and residual error (denoted as 'ERR') defined by the following.

$$
E R R:=\left\|\mathcal{A} x_{k}^{m-1}-\left|x_{k}\right|^{[m-1]}-b\right\| .
$$

All computations are started from $x_{(0)}=0$ and $y_{(0)}=0$ and terminated once ERR at the current iterative $x_{(k)} \leq 10^{-10}$ or the maximal number of iterative $k_{\text {max }}$ exceeds 1000 . 
In what follows, we consider the tensor preconditioned splitting of (10):

$$
P \cdot \mathcal{A}=\hat{\mathcal{A}}=\hat{\mathcal{A}}_{D}-\hat{\mathcal{A}}_{L}-\hat{\mathcal{A}}_{U},
$$

where $\hat{\mathcal{A}}_{D}=A_{D} \mathcal{I}_{m}, \hat{\mathcal{A}}_{L}=A_{L} \mathcal{I}_{m}, \hat{\mathcal{A}}_{U}=A_{U} \mathcal{I}_{m}$, and $A_{D},-A_{L},-A_{U}$ are the diagonal parts, strictly lower and strictly upper triangle part of $M(P \cdot \mathcal{A})$ and $\mathcal{I}_{m}$ is a unit tensor as previously mentioned.

A type of preconditioner (as a variant form in [40]) $P_{\alpha}=I+S_{\alpha}$ is considered, where the following is the case.

$$
S_{\alpha}=\left(\begin{array}{ccccc}
0 & -\alpha_{1} a_{12 \cdots 2} & 0 & \cdots & 0 \\
-\alpha_{1} a_{21 \cdots 1} & 0 & -\alpha_{2} a_{23 \cdots 3} & \cdots & 0 \\
\vdots & \ddots & \ddots & \ddots & 0 \\
0 & 0 & \ddots & \ddots & -\alpha_{n-1} a_{n-1, n \cdots n} \\
0 & 0 & 0 & -\alpha_{n-1} a_{n, n-1 \cdots n-1} & 0
\end{array}\right) \text {, }
$$

$\alpha_{i}=0.01, i=1,2, \cdots n-1 . I$ is an identity matrix with appropriate dimension.

All numerical experiments have been carried out by MATLAB R2011b 7.1.3 on a PC equipped with an Intel(R) Core(TM) i7-2670QM, CPU running at 2.20GHZ with 8 GB of RAM in Windows 7 operating system.

Example 1. First, consider the tensor absolute Equation (10) with a strong $\mathcal{M}$-tensor $\mathcal{A}$ in different cases.

Case $1 . \mathcal{A}=\eta \mathcal{I}_{3}-\mathcal{B}$, where $\mathcal{B} \in \mathbb{R}^{[3, n]}$ is a non-negative tensor with $b_{i_{1}, i_{2}, i_{3}}=\mid \tan \left(i_{1}+\right.$ $\left.i_{2}+i_{3}\right) \mid$.

Case 2. $\mathcal{A}=n^{3} \mathcal{I}_{3}-\mathcal{B}$, where $\mathcal{B} \in \mathbb{R}^{[3, n]}$ is a non-negative tensor with $b_{i_{1}, i_{2}, i_{3}}=\mid \sin \left(i_{1}+\right.$ $\left.i_{2}+i_{3}\right) \mid$.

Case 3. $\mathcal{A}=s \mathcal{I}_{3}-\mathcal{B}$, where $\mathcal{B} \in \mathbb{R}^{[3, n]}$ is generated randomly by MATLAB, and $s=$ $(1+\delta) \max _{1,2, \cdots, n}\left(\mathcal{B}^{2}\right)_{i}, \mathbf{e}=(1,1, \cdots, 1)^{T}$.

We provide three different cases for different tensors $\mathcal{A}$ and $\mathcal{B}$ with various sizes. Parameters $\sigma, \omega, \eta, \delta$ and sizes $n$ are concretely shown in Tables or Figures.

The numerical results have been shown in Tables 1-3 and Figures 1 and 2. From the numerical results, we can see that TAOR and TILM are all efficient methods. Case 3 seems to be the best condition. From an iterative parameter point of view in experiments, a better choice of $\sigma$ and $\omega$ may be close to 1.2. It is observed that once parameters $\sigma$ and $\omega$ are selected as 1, TAOR will reduce to TSOR, which is displayed in line 4 in Tables 1 and 2. By all performances of convergence, TSOR is also a quite efficient approach. However, TAOR seems to be a more efficient method than TILM and TSOR methods from all aspects due to the flexible selection of parameters. In particular, in the aspects of IT number and elapsed CPU, TAOR is superior to TILM distinctly, despite these ERRs of the latter descending rapidly in the last few steps. Furthermore, from the residual trend chart with the changing numbers of iteration in Figures 1 and 2, we can find the desired performances of the established methods. 


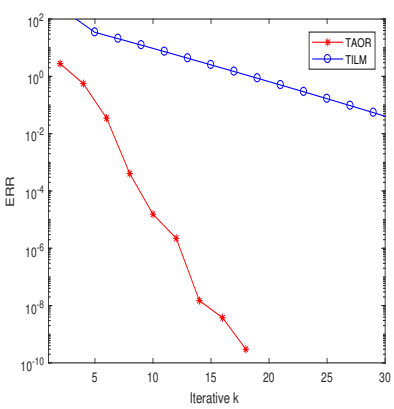

(a) case $1, \eta=800$

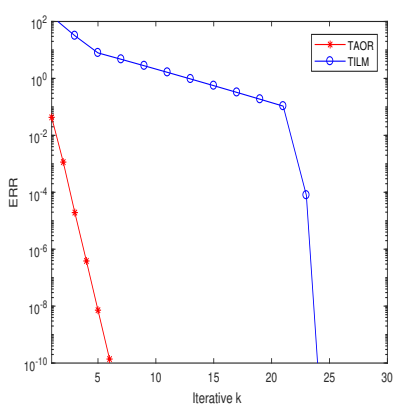

(b) case2

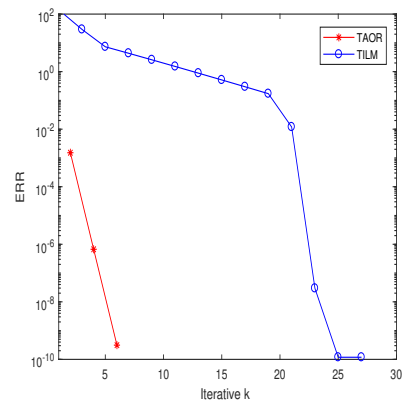

(c) case $3, \delta=8$.

Figure 1. The residual ERR for TAOR and TILM methods with different cases in Example 1, $\sigma=1.1$, $\omega=1.3, n=5$

Table 1. Preconditioned numerical results for Example 1, $n=5$.

\begin{tabular}{|c|c|c|c|c|}
\hline Case & & 1 & 2 & 3 \\
\hline \multirow{3}{*}{$\begin{array}{c}\mathrm{TAOR}, \sigma=1.1 \\
\omega=1.3\end{array}$} & It & 18 & 6 & 5 \\
\hline & $\mathrm{CPU}$ & 0.1413 & 0.1118 & 0.0743 \\
\hline & RES & $3.2455 \times 10^{-11}$ & $2.6210 \times 10^{-12}$ & $6.7591 \times 10^{-12}$ \\
\hline \multirow{3}{*}{$\begin{array}{c}\text { TAOR } \sigma=0.1 \\
\omega=1.1\end{array}$} & It & 15 & 5 & 5 \\
\hline & $\mathrm{CPU}$ & 0.1215 & 0.1098 & 0.0713 \\
\hline & RES & $2.8875 \times 10^{-11}$ & $9.1778 \times 10^{-13}$ & $3.9930 \times 10^{-13}$ \\
\hline \multirow{3}{*}{$\begin{array}{c}\text { TSOR, } \sigma=1.0 \\
\omega=1.0\end{array}$} & It & 20 & 6 & 5 \\
\hline & CPU & 0.1662 & 0.1098 & 0.0914 \\
\hline & RES & $4.0899 \times 10^{-11}$ & $1.8440 \times 10^{-12}$ & $6.6225 \times 10^{-12}$ \\
\hline \multirow{3}{*}{ TILM } & It & 34 & 25 & 28 \\
\hline & CPU & 0.4920 & 0.3498 & 0.4861 \\
\hline & RES & $1.4108 \times 10^{-11}$ & $5.5511 \times 10^{-12}$ & $1.1809 \times 10^{-12}$ \\
\hline
\end{tabular}

Table 2. Preconditioned numerical results for Example 1, $n=10$.

\begin{tabular}{|c|c|c|c|c|}
\hline Case & & 1 & 2 & 3 \\
\hline \multirow{3}{*}{$\begin{array}{c}\text { TAOR }, \sigma=1.2, \\
\omega=1.2\end{array}$} & It & 10 & 4 & 6 \\
\hline & CPU & 0.1295 & 0.1223 & 0.0807 \\
\hline & RES & $1.4571 \times 10^{-12}$ & $6.0335 \times 10^{-14}$ & $4.2387 \times 10^{-12}$ \\
\hline \multirow{3}{*}{$\begin{array}{c}\text { TAOR }, \sigma=0.2, \\
\omega=1.3\end{array}$} & It & 10 & 10 & 9 \\
\hline & CPU & 0.1894 & 0.1466 & 0.0960 \\
\hline & RES & $1.4571 \times 10^{-12}$ & $6.7092 \times 10^{-12}$ & $1.9524 \times 10^{-11}$ \\
\hline \multirow{3}{*}{$\begin{array}{c}\text { TSOR }, \sigma=1.0 \\
\omega=1.0\end{array}$} & It & 10 & 5 & 5 \\
\hline & CPU & 0.1503 & 0.1098 & 0.0914 \\
\hline & RES & $1.4571 \times 10^{-12}$ & $7.0335 \times 10^{-14}$ & $6.6225 \times 10^{-12}$ \\
\hline \multirow{3}{*}{ TILM } & It & 45 & 43 & 35 \\
\hline & CPU & 0.6000 & 0.5883 & 0.5320 \\
\hline & RES & $7.1598 \times 10^{-11}$ & $6.8692 \times 10^{-16}$ & $4.9104 \times 10^{-13}$ \\
\hline
\end{tabular}


Table 3. Preconditioned numerical results for Example 1, $n=50$.

\begin{tabular}{ccccc}
\hline Case & & $\mathbf{1}$ & $\mathbf{2}$ & $\mathbf{3}$ \\
\hline \multirow{2}{*}{ TAOR, $\sigma=0.8}$, & It & 13 & 6 & 8 \\
$\omega=1.3$ & CPU & 0.4183 & 0.3241 & 0.2626 \\
& RES & $1.6573 \times 10^{-11}$ & $7.0635 \times 10^{-13}$ & $5.2853 \times 10^{-11}$ \\
\hline \multirow{2}{*}{ TSOR, $\sigma=0.8}$, & It & 13 & 7 & 9 \\
$\omega=0.8$ & CPU & 0.6902 & 0.5926 & 0.4531 \\
& RES & $1.5732 \times 10^{-11}$ & $8.4235 \times 10^{-13}$ & $6.8265 \times 10^{-11}$ \\
\hline & It & 48 & 46 & 39 \\
TILM & CPU & 1.835 & 1.3452 & 1.2921 \\
& RES & $8.1845 \times 10^{-10}$ & $7.9655 \times 10^{-13}$ & $4.9104 \times 10^{-11}$ \\
\hline
\end{tabular}

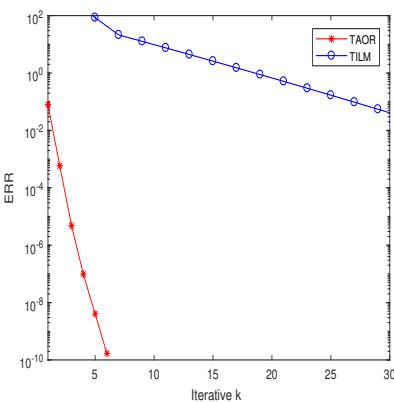

(a) case $1, \eta=3000$

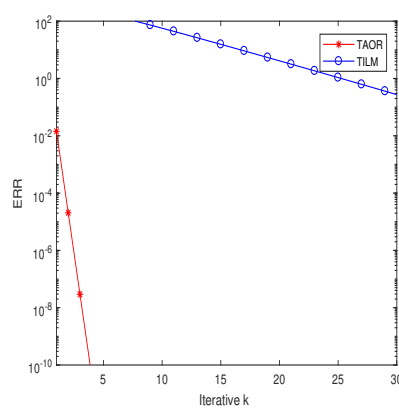

(b) case2

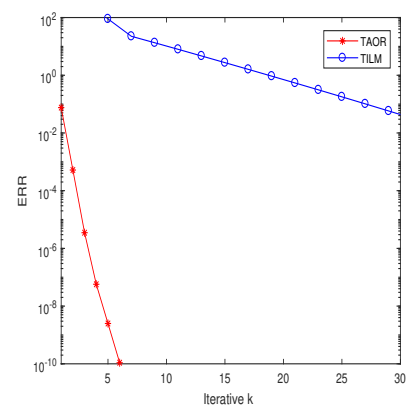

(c) case $3, \delta=8$.

Figure 2. The residual ERR for TAOR and TILM methods with difference cases in Example 1, $\sigma=1.2$, $\omega=1.2$ and $n=10$.

Example 2. Consider the following TAVE:

$$
\mathcal{A} x^{m-1}-|x|^{[m-1]}=b,
$$

where $\mathcal{A}=2 * \mathcal{I}_{m}-\vartheta * B$

$$
\begin{gathered}
\mathcal{B}(:,:, 1)=\left(\begin{array}{ccc}
0.580 & 0.2432 & 0.1429 \\
0 & 0.4109 & 0.0701 \\
0.4190 & 0.3459 & 0.7870
\end{array}\right), \quad \mathcal{B}(:,:, 2)=\left(\begin{array}{lll}
0.4708 & 0.1330 & 0.0327 \\
0.1341 & 0.5450 & 0.2042 \\
0.3951 & 0.3220 & 0.7631
\end{array}\right), \\
\mathcal{B}(:,:, 3)=\left(\begin{array}{lll}
0.4381 & 0.1003 & 0 \\
0.0229 & 0.4338 & 0.0930 \\
0.5390 & 0.4659 & 0.9070
\end{array}\right),
\end{gathered}
$$

$\mathcal{I}_{m}$ is an identity tensor of order 3 dimension 3 . In this example, we set $x_{*}=(-1,1,-1)^{T}$. Then, it generates the right hand $b$.

In this example, we discuss the probable optimal parameters of TAOR. In order to guarantee the strong $\mathcal{M}$-tensor of $\mathcal{A}$, set $\vartheta=0.1$. All numerical results are depicted in Figures 3-6.

From Figures 3-6, we find that when parameter $\omega$ is fixed on interval [0.8-1.2], the other parameter $\sigma$ shows better performance nearby 1.2 or 1.3. IT and CPU seem to have similar trends in their process of variation. Note that when $\omega$ is selected with 1.2, the changes of IT and CPU tend to be stable between 0.4 and 1.6 for parameter $\sigma$. These results show a similar trend with Example 1. 


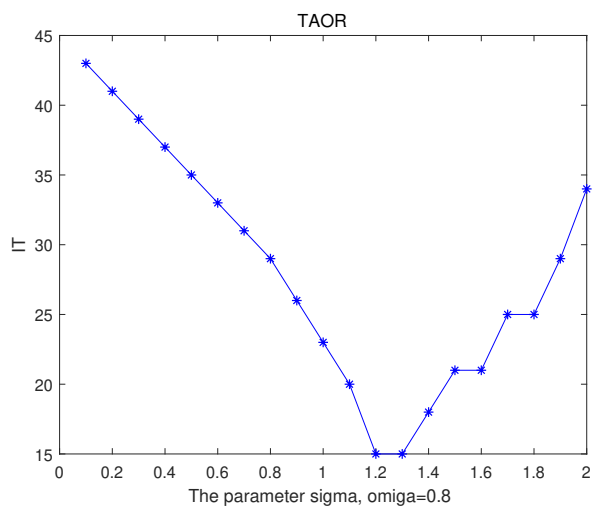

(a) IT versus parameter $\sigma$

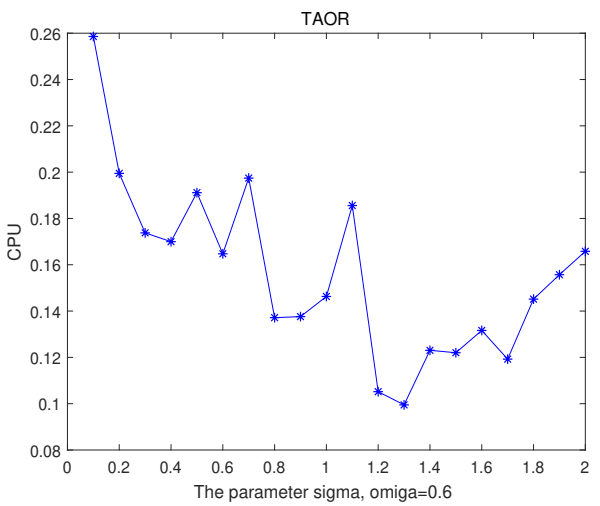

(b) CPU versus parameter $\sigma$

Figure 3. IT (a) and CPU (b) versus parameters $\sigma, \omega=0.6$ in Example 2.

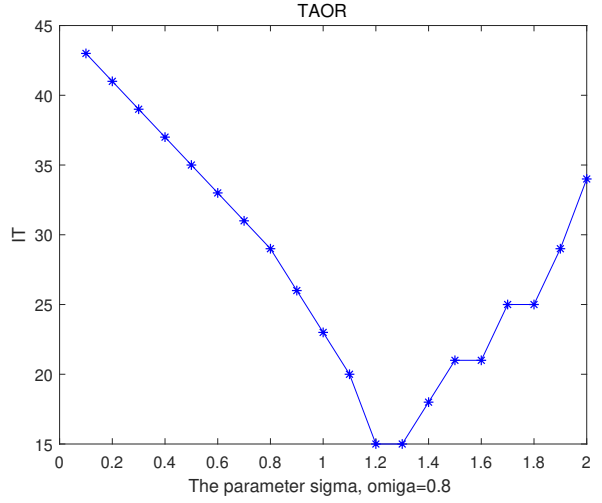

(a) IT versus parameter $\sigma$

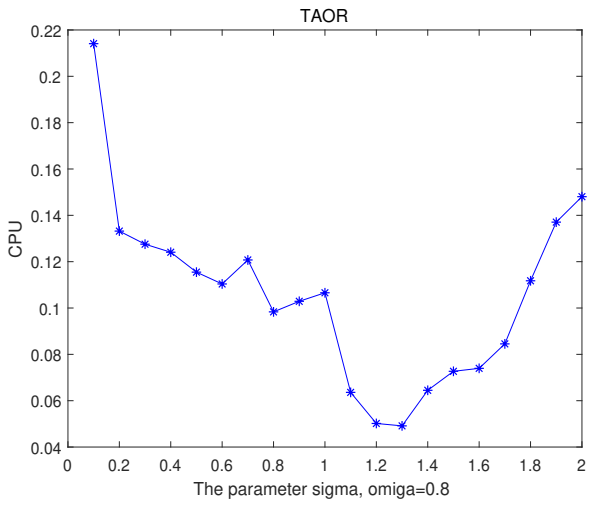

(b) CPU versus parameter $\sigma$

Figure 4. IT (a) and CPU (b) versus parameters $\sigma, \omega=0.8$ in Example 2.

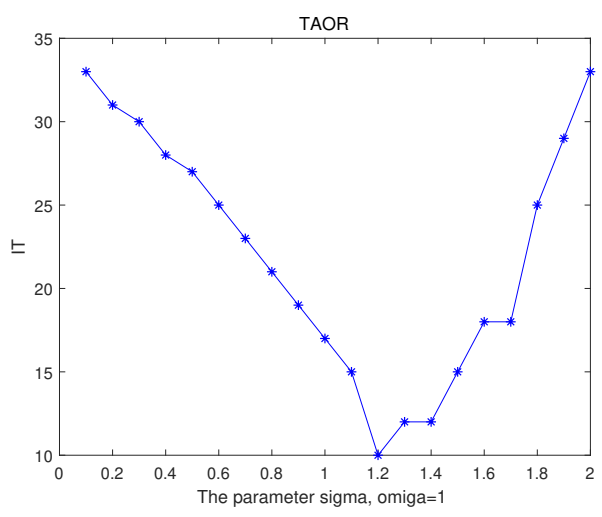

(a) IT versus parameter $\sigma$

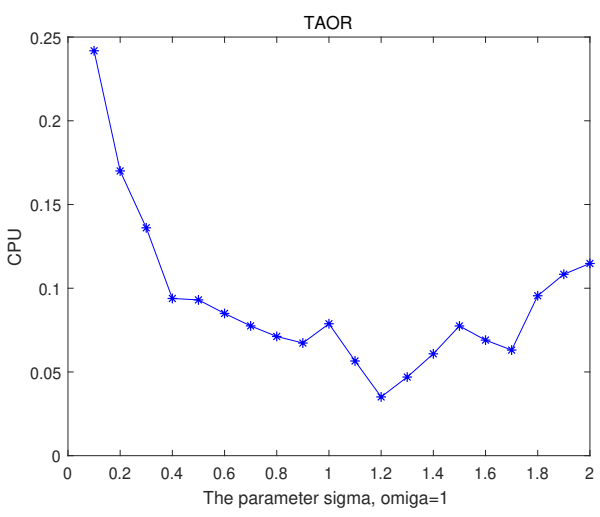

(b) CPU versus parameter $\sigma$

Figure 5. IT (a) and CPU (b) versus parameters $\sigma, \omega=1.0$ in Example 2. 


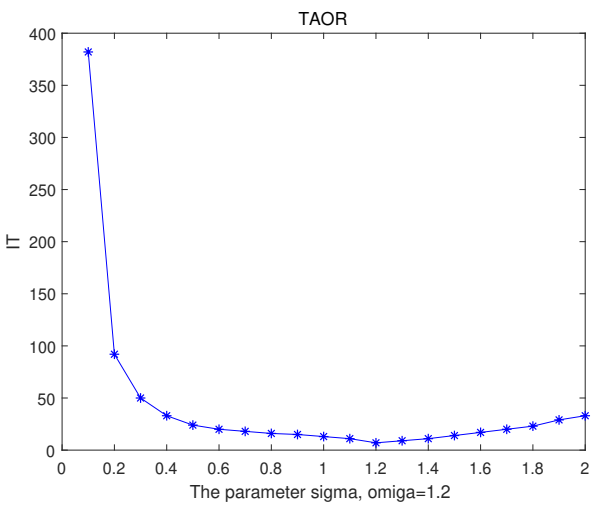

(a) IT versus parameter $\sigma$

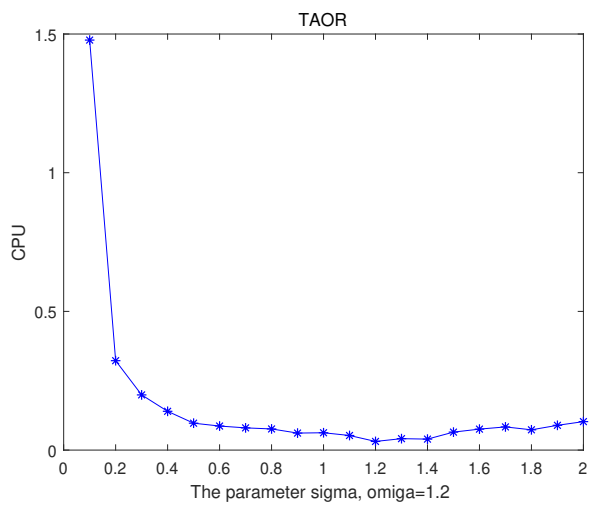

(b) CPU versus parameter $\sigma$

Figure 6. IT (a) and CPU (b) versus parameters $\sigma, \omega=1.2$ in Example 2.

\section{Conclusions}

In this paper, tensor splitting iteration schemes TAOR and TSOR are proposed for solving the tensor absolute value equation (TAVE), which can be regarded as generalizations of AOR and SAOR for linear systems. Some efficient preconditioned techniques are provided to improve the efficiency of solution-finding of TAVE. Meanwhile, we attempt to provide probability selections of optimal parameters from the perspective of tentative examples. The proposed approaches have been demonstrated to be superior to the existing method (rare at present) under some conditions, which can be fully validated in our numerical experiments section. Theoretical optimal parameters will be the considerations of our future research work.

Author Contributions: All authors contributed equally and significantly in writing this article. All authors have read and agreed to the published version of the manuscript.

Funding: The project is supported by Fujian Natural Science Foundation (Grant No. 2019J01879) and Key Reform in Education (Grant No. FBJG20200310).

Institutional Review Board Statement: Not applicable.

Informed Consent Statement: Not applicable.

Conflicts of Interest: The authors declare that there is no conflict of interests regarding the publication of this article.

\section{References}

1. Cipolla, S.; Redivo-Zaglia, M.; Tudisco, F. Extrapolation methods for fixed-point multilinear PageRank computations. Numer. Linear Algebra Appl. 2020, 27, e2280. [CrossRef]

2. He, H.; Ling, C.; Qi, L.; Zhou, G. A Globally and Quadratically Convergent Algorithm for Solving Multilinear Systems with $\mathcal{M}$-tensors. J. Sci. Comput. 2018, 76, 1718-1741. [CrossRef]

3. Lv, C.Q.; Ma, C.F. A Levenberg-Marquardt method for solving semi-symmetric tensor equations. J. Comput. Appl. Math. 2018, 332, 13-25. [CrossRef]

4. Wang, X.; Che, M.L.; Wei, Y.M. Neural networks based approach solving multi-linear systems with $\mathcal{M}$-tensors. Neurocomputing 2019, 351, 33-42. [CrossRef]

5. Xie, Z.J.; Jin, X.Q.; Wei, Y.M. Tensor ethods for Solving Symmetric $\mathcal{M}$-tensor Systems. J. Sci. Comput. 2018, 74, 412-425. [CrossRef]

6. Xie, Z.J.; Jin, X.Q.; Wei, Y.M. A fast algorithm for solving circulant tensor systems. Linear Multilinear Algebra 2017, 65, 1894-1904. [CrossRef]

7. El Ichi, A.; Jbilou, K.; Sadaka, R. Tensor Global Extrapolation Methods Using the n-Mode and the Einstein Products. Mathematics 2020, 8, 1298. [CrossRef]

8. Kolda, T.G. Multilinear Operators for Higher-Order Decompositions; Technical Report SAND2006-2081; Sandia National Laboratories: Albuquerque, NM, USA; Livermore, CA, USA, 2006.

9. Liu, D.D.; Li, W.; Vong, S.W. Relaxation methods for solving the tensor equation arising from the higher-order Markov chains. Numer. Linear Algebra Appl. 2019, 25, e2260. [CrossRef] 
10. Heyouni, M.; Saberi-Movahed, F.; Tajaddini, A. A tensor format for the generalized Hessenberg method for solving Sylvester tensor equations. J. Comput. Appl. Math. 2020, 377, 112878. [CrossRef]

11. Reichel, L.; Ugwu, U.O. Tensor Arnoldi-Tikhonov and GMRES-Type Methods for Ill-Posed Problems with a t-Product Structure. J. Sci. Comput. 2022, 90, 1-39. [CrossRef]

12. Saberi-Movahed, F.; Tajaddini, A.; Heyouni, M.; Elbouyahyaoui, L. Some iterative approaches for Sylvester tensor equations, Part I: A tensor format of truncated Loose Simpler GMRES. Appl. Numer. Math. 2022, 172, 428-445. [CrossRef]

13. Song, Y.S.; Qi, L.Q. Spectral properties of positively homogeneous operators induced by higher order tensors. SIAM J. Matrix Anal. Appl. 2013, 34, 1581-1595. [CrossRef]

14. Xie, Y.J.; Ke, Y.F. Neural network approaches based on new NCP-functions for solving tensor complementarity problem. J. Appl. Math. Comput. 2021, 4, 1-21. [CrossRef]

15. Zhang, L.P.; Qi, L.Q.; Zhou, G.L. M-tensors and some applications. SIAM J. Matrix Anal. Appl. 2014, 35, 437-452. [CrossRef]

16. Song, Y.S.; Qi, L.Q. Properties of some classes of structured tensors. J. Optim. Theory Appl. 2015, 165, 854-873. [CrossRef]

17. Ke, Y.F.; Ma, C.F. SOR-like iteration method for solving absolute value equations. Appl. Math. Comput. 2017, 311, 195-202. [CrossRef]

18. Mangasarian, O.L.; Meyer, R.R. Absolute value equations. Linear Algebra Appl. 2006, 419, 359-367. [CrossRef]

19. Che, M.L.; Qi, L.Q.; Wei, Y.M. Positive definite tensors to nonlinear complementarity problems. J. Optim. Theory Appl. 2016, 168, 475-487. [CrossRef]

20. Chen, L.; Qi, L. A semismooth Newton method for tensor eigenvalue complementarity problem. Comput. Optim. Appl. 2016, 65, 109-126. [CrossRef]

21. Ding, W.Y.; Wei, Y.M. Solving multilinear systems with $\mathcal{M}$-tensors. J. Sci. Comput. 2016, 68, 689-715. [CrossRef]

22. Ding, W.Y.; Wei, Y.M. Generalized tensor eigenvalue problems. SIAM J. Matrix Anal. Appl. 2015, 36, 1073-1099. [CrossRef]

23. Han, L.X. A homotopy method for solving multilinear systems with $\mathcal{M}$-tensors. Appl. Math. Lett. 2017, 69, 49-54. [CrossRef]

24. Liu, D.D.; Li, W.; Vong, S.W. The tensor splitting with application to solve multi-linear systems. J. Comput. Appl. Math. 2018, 330, 75-94. [CrossRef]

25. Li, W.; Liu, D.D.; Vong, S.W. Comparison results for splitting iterations for solving multi-linear systems. Appl. Numer. Math. 2018, 134, 105-121. [CrossRef]

26. Li, W.; Ng, M.K. On the limiting probability distribution of a transition probability tensor. Linear Multilinear Algebra 2014, 62, 362-385. [CrossRef]

27. Du, S.; Zhang, L.; Chen, C.; Qi, L. Tensor absolute value equations. Sci. China Math. 2018, 61, 1695-1710. [CrossRef]

28. Ling, C.; Yan, W.; He, H.; Qi, L. Further study on tensor absolute value equations. Sci. China Math. 2019, 63, 2137-2156. [CrossRef]

29. Matveev, S.A.; Zheltkov, D.A.; Tyrtyshnikov, E.E.; Smirnov, A.P. Tensor train versus Monte Carlo for the multicomponent Smoluchowski coagulation equation. J. Comput. Phys. 2016, 316, 164-179. [CrossRef]

30. Smirnov, A.P.; Matveev, S.A.; Zheltkov, D.A.; Tyrtyshnikov, E.E. Fast and accurate finite-difference method solving multicomponent Smoluchowski coagulation equation with source and sink terms. Procedia Comput. Sci. 2016, 80, 2141-2146. [CrossRef]

31. Jiang, Z.; Li, J. Solving tensor absolute value equation. Appl. Numer. Math. 2021, 170, 255-268. [CrossRef]

32. Zheltkov, D.; Tyrtyshnikov, E. Global optimization based on TT-decomposition. Russ. J. Numer. Anal. Math. Model. 2020, 35, 247-261. [CrossRef]

33. Cichocki, A.; Zdunek, R.; Phan, A.H.; Amari, S.I. Nonnegative Matrix and Tensor Factorizations: Applications to Exploratory Multi-Way Data Analysis and Blind Source Separation; John Wiley \& Sons: Hoboken, NJ, USA, 2009.

34. Kolda, T.G.; Bader, B.W. Tensor decompositions and applications. SIAM Rev. 2009, 51, 455-500. [CrossRef]

35. Pearson, K. Essentially positive tensors. Int. J. Algebra 2010, 4, 421-427.

36. Qi, L.Q. Eigenvalues of a real supersymmetric tensor. J. Symb. Comput. 2005, 40, 1302-1324. [CrossRef]

37. Che, M.L.; Qi, L.Q.; Wei, Y.M. M-tensors and nonsingular $\mathcal{M}$-tensors. Linear Algebra Appl. 2013, 439, $3264-3278$.

38. Li, D.H.; Xie, S.; Xu, R.H. Splitting methods for tensor equations. Numer. Linear Algebra Appl. 2017, 24, 5e2102. [CrossRef]

39. Qi, L.Q.; Luo, Z. Tensor Analysis: Spectral Theory and Special Tensors; Society for Industrial and Applied Mathematics: Philadelphia, PA, USA, 2017.

40. Kohno, T.; Kotakemori, H.; Niki, H.; Usui, M. Improving the modified Gauss-Seidel method for Z-matrices. Linear Algebra Appl. 1997, 267, 113-123. [CrossRef] 